\title{
Structural origins of the boson peak in metals: From high-entropy alloys to metallic glasses
}

\author{
Tobias Brink, ${ }^{*}$ Leonie Koch, and Karsten Albe \\ Fachgebiet Materialmodellierung, Institut für Materialwissenschaft, \\ TU Darmstadt, Jovanka-Bontschits-Straße 2, D-64287 Darmstadt, Germany
}

(Dated: December 14th, 2016)

\begin{abstract}
The boson peak appears in all amorphous solids and is an excess of vibrational states at low frequencies compared to the phonon spectrum of the corresponding crystal. Until recently, the consensus was that it originated from "defects" in the glass. The nature of these defects is still under discussion, but the picture of regions with locally disturbed short-range order and/or decreased elastic constants has gained some traction. Recently, a different theory was proposed: The boson peak was attributed to the first van Hove singularity of crystal lattices which is only smeared out by the disorder. This new viewpoint assumes that the van Hove singularity is simply shifted by the decreased density of the amorphous state and is therefore not a glass-specific anomaly. In order to resolve this issue, we use computer models of a four-component alloy, alternatively with chemical disorder (high-entropy alloy), structural disorder, and reduced density. Comparison to a reference glass of the same composition reveals that the boson peak consists of additional vibrational modes which can be induced solely by structural disorder. While chemical disorder introduces fluctuations of the elastic constants, we find that those do not lead to sufficient local softening to induce these modes. A boson peak due to a reduction of density could be excluded for the present metallic system.
\end{abstract}

Published in:

T. Brink et al., Phys. Rev. B 94, 224203 (2016)

(C) 2016 American Physical Society.

\section{INTRODUCTION}

Amorphous materials show an excess contribution in the terahertz region of the vibrational density of states (VDOS) as compared to the corresponding crystal or the Debye model. The origin of this so-called boson peak is still a matter of ongoing discussion. Early explanations include scattering of phonons at density fluctuations [1] and the soft potential model [2-6]. The latter assumes an interaction of acoustic phonons with quasi-localized modes arising from "defects" in the disordered structure [7-9]. It was proposed that these defects are loosely packed atoms $[6,10-13]$ or resemble interstitialcies $[14,15]$. An alternative explanation, where the boson peak is considered to be a precursor of a dynamical instability, was brought forward by Grigera et al. [16], while Schirmacher and colleagues developed a theory where fluctuating force or elastic constants give rise to the additional modes that make up the boson peak [17-22]. This viewpoint is supported by studies that connect the boson peak to "soft spots," i.e., regions of reduced short-range order, stiffness, and mechanical strength [13, 23-25]. For silicon-based glasses, similar findings exist $[26,27]$ and a relation between the bending rigidity of the silicon tetrahedra, the shear modulus, and the boson peak has been proposed [28].

In contrast to these theories, which describe the boson peak as additional modes present only in glasses, Taraskin et al. proposed that the boson peak is related to the first van Hove singularity of the crystal, which is shifted to lower frequencies by fluctuating force constants [29]. More recently, work by Chumakov and colleagues re- ceived attention [30-34]: Measurements on oxide glasses suggest that the boson peak is simply a van Hove singularity that is shifted because of the lower volume density of the glass compared to the crystal. These authors criticize alternative models like Schirmacher's as they do not explicitly treat the (pseudo-)Brillouin zone and therefore do not include the necessary van Hove singularities [33]. Furthermore, the argument is that disordered systems are always less dense than ordered ones. That leads to a lower transversal sound velocity, to a smaller size of the (pseudo-)Brillouin zone, and in consequence to a shift to lower frequencies, the result of which was interpreted as a boson peak [33]. This would equally apply to soft spots, which are also characterized by a lowered density.

A material class carrying all the ingredients of those models arguing for a boson peak induced by fluctuations in the elastic medium, while still being crystalline and similar in density to the crystalline forms of the constituent elements, are high-entropy alloys (HEAs). HEAs are metallic alloys consisting of at least four elements with equal or near equal molar fractions that are considered to be thermodynamically stable phases because of their large configurational entropy [35-37]. Single-phase HEAs are random solid solutions and as such-from the point of view of lattice dynamics - they can be thought of as a simple crystal lattice with randomly distributed force constants. Therefore, they are a suitable model to discern between the different proposed origins of the boson peak. Experimentally, though, HEAs have been mostly studied with respect to their mechanical and thermodynamic properties, but not their vibrational properties. We use molecular dynamics (MD) simulations on a CuNiCoFe al- 
loy, since MD provides direct access to vibrational properties and furthermore allows for additional modifications of the HEA model: Elastic scaling of the volume of the crystalline matrix, as well as the gradual introduction of disorder are possible. For the introduction of disorder, we follow the ideas of interstitialcy theory which proposes that the melting of metals is comparable to the introduction of increasing numbers of interstitial defects that remain discernible even in the liquid and glassy states [38]. Using this tunable model, and by comparison with a metallic glass of the same composition - quenched from the melt at high cooling rates-, we can identify the origin of the boson peak.

\section{SIMULATION METHODS AND ANALYSIS}

\section{A. Preparation of CuNiCoFe high-entropy alloys and glasses}

We use MD simulations on a $\mathrm{CuNiCoFe}$ alloy consisting of $N=102816$ atoms with an embedded atom method potential by Zhou et al. [39]. This provides a realistic description of the vibrational properties but does not contain electronic or magnetic effects, which we can safely ignore in discussing the boson peak. All simulations were performed with LAMMPS [40]. In order to generate the initial HEA structure, we used a hybrid Monte Carlo/MD method in the variance-constrained semi-grand-canonical ensemble [41], which provides a significant reduction of computation time needed to reach the equilibrium chemical order. Here, we performed $N / 4$ Monte Carlo trial moves in between every $20 \mathrm{MD}$ steps at $800 \mathrm{~K}$. The constraint on the variation of the elemental concentrations allows one to study phase formation - even in miscibility gaps - and simultaneously maintain an equimolar composition [41]. Since element concentrations depend on the excess chemical potentials $\Delta \mu_{i}$, the simulation needs a set of chemical potential differences $\left\{\Delta \mu_{i}\right\}$ as input. If a set $\left\{\Delta \mu_{i}\right\}$ exists, such that the system is still miscible in a random solid solution, the resulting sample is a good model for a single-phase HEA. To probe for the chemical potential differences, we disabled the variance constraint and employed Gaussian processes [42], a global optimization algorithm: We used $\left\{\Delta \mu_{i}\right\}$ as the parameters and minimized the deviation from equimolar concentrations obtained by running simulations in the semigrand-canonical ensemble. This works because without the variance constraint an immiscible system will reduce the concentration of one or more elements, while a miscible system will stay equimolar. We found a solution in which the system is in fact miscible: The final chemical potential differences relative to $\mathrm{Cu}$ are $\Delta \mu_{\mathrm{Cu}-\mathrm{Ni}}=0.9 \mathrm{eV}$, $\Delta \mu_{\mathrm{Cu}-\mathrm{Co}}=0.85 \mathrm{eV}$, and $\Delta \mu_{\mathrm{Cu}-\mathrm{Fe}}=0.7 \mathrm{eV}$.

We performed this investigation at $800 \mathrm{~K}$ because this makes it easier to find a miscible alloy: The $T S$-term due to chemical disorder dominates the thermodynamics of the system. This is a reasonable facsimile of the ex- perimental synthesis, which often starts from the melt. If this alloy, and HEAs in general, are still miscible at lower temperatures - or if they are simply kinetically trapped during quenching - is an important unsolved question which lies beyond the scope of this work.

In addition to the HEA sample, we also prepared a glass sample of the same composition by quenching from the melt at $2000 \mathrm{~K}$ to $30 \mathrm{~K}$ with a cooling rate of $10^{13} \mathrm{~K} / \mathrm{s}$ (the minimum rate at which crystallization is still kinetically suppressed).

\section{B. Calculation of the vibrational density of states}

We measured the VDOS by recording the velocity autocorrelation function during an MD run, the Fourier transform of which is the density of states $g(\nu)$ [43]. All systems were first equilibrated for $0.5 \mathrm{~ns}$ at $30 \mathrm{~K}$ and ambient pressure; the velocity auto-correlation function was subsequently measured at $30 \mathrm{~K}$ in the micro-canonical ensemble. We use the average of 100 auto-correlation functions to reduce the noise. To differentiate the contributions of different structures in the system, we also present data from partial VDOS calculations. Here, we consider only the partial velocity auto-correlation function of the atoms of interest to calculate the VDOS. While one could argue that phonons are delocalized over the whole system, computer simulations using similar schemes [12] evidence the effectiveness of this method in the case of the boson peak: Its modes seem to be localized enough to be able to separate them out by spatial sampling of the VDOS.

Thermodynamic properties for the low-temperature regime - such as the internal energy $U$ and the heat capacity $C$-were derived from the VDOS via the harmonic approximation [44]

$$
\begin{aligned}
& U=\frac{3}{2} N \int_{0}^{\infty} h \nu \operatorname{coth}\left(\frac{1}{2} \frac{h \nu}{k_{\mathrm{B}} T}\right) g(\nu) \mathrm{d} \nu \text { and } \\
& C=3 N k_{\mathrm{B}} \int_{0}^{\infty}\left(\frac{h \nu}{2 k_{\mathrm{B}} T}\right)^{2} \sinh ^{-2}\left(\frac{h \nu}{2 k_{\mathrm{B}} T}\right) g(\nu) \mathrm{d} \nu .
\end{aligned}
$$

The Debye temperature $\Theta$ and frequency $\nu_{\mathrm{D}}$ can then be obtained from the zero point energy via [44]

$$
U_{0}=\frac{9}{8} N k_{\mathrm{B}} \Theta=\frac{9}{8} N h \nu_{\mathrm{D}} .
$$

Given the condition of $\int_{0}^{\nu_{\mathrm{D}}} g_{\mathrm{D}}(\nu) \mathrm{d} \nu=1$, one can obtain the complete VDOS in the Debye model from $\nu_{\mathrm{D}}$.

\section{Calculation of the shear modulus}

In order to probe the local stiffness of our samples, we calculated the $0 \mathrm{~K}$ stiffness tensor for every atom. This was done using molecular statics simulations in which we subsequently impose the stress components $\sigma_{x x}, \sigma_{y y}, \sigma_{z z}$, $\sigma_{x y}, \sigma_{x z}$, and $\sigma_{y z}$. For this, the currently active component $\sigma_{i}$ was set to $50 \mathrm{MPa}$ and all other components $\sigma_{j \neq i}$ 
were set to zero. After static minimization, we calculate the resulting per-atom strain tensors using the atomic strain calculation in ovito $[45,46]$. Using Hooke's law in tensor form (Voigt notation),

$$
s_{i j}=\frac{\varepsilon_{i}}{\sigma_{j}}
$$

we obtain the compliance tensor $s$. The stiffness tensor $c$ is simply its inverse. It should be noted that this method for the calculation of elastic constants per atom can fail in the case of microscopic plastic events. We mostly avoid this by using a low stress value, but for a small number of atoms the stiffness may be unrealistically high or low. The results we present later evidence that this method is reliable enough for the present purpose.

While an amorphous system should have an isotropic shear modulus, (partially) crystalline samples show a large anisotropy. Earlier work by Derlet and colleagues [24] demonstrated that the five eigenshear moduli obtained from a Kelvin stiffness tensor [47] are appropriate to characterize the elastic moduli of amorphous systems. The Voigt notation of the stiffness tensor can easily be converted to the Kelvin notation by the element-wise multiplication

$$
c_{i j}^{\text {Kelvin }}=A_{i j} c_{i j}^{\text {Voigt }}
$$

with

$$
A=\left(\begin{array}{cccccc}
1 & 1 & 1 & \sqrt{2} & \sqrt{2} & \sqrt{2} \\
1 & 1 & 1 & \sqrt{2} & \sqrt{2} & \sqrt{2} \\
1 & 1 & 1 & \sqrt{2} & \sqrt{2} & \sqrt{2} \\
\sqrt{2} & \sqrt{2} & \sqrt{2} & 2 & 2 & 2 \\
\sqrt{2} & \sqrt{2} & \sqrt{2} & 2 & 2 & 2 \\
\sqrt{2} & \sqrt{2} & \sqrt{2} & 2 & 2 & 2
\end{array}\right)
$$

We follow Derlet et al. in calculating the shear moduli by first projecting out the volume changes [24]:

$$
c^{\prime}=P^{\mathrm{T}} \cdot c^{\text {Kelvin }} \cdot P
$$

with

$$
P=\left(\begin{array}{cccccc}
+\frac{2}{3} & -\frac{1}{3} & -\frac{1}{3} & 0 & 0 & 0 \\
-\frac{1}{3} & +\frac{2}{3} & -\frac{1}{3} & 0 & 0 & 0 \\
-\frac{1}{3} & -\frac{1}{3} & +\frac{2}{3} & 0 & 0 & 0 \\
0 & 0 & 0 & 1 & 0 & 0 \\
0 & 0 & 0 & 0 & 1 & 0 \\
0 & 0 & 0 & 0 & 0 & 1
\end{array}\right)
$$

The shear moduli are now the five eigenvalues of $c^{\prime}$, which-in an isotropic material-correspond to two times the usual shear modulus definition. A softening of the lowest shear modulus $\left(G_{1}\right)$ was connected to the boson peak modes [24], which is why we chose this value as our characteristic elastic constant.

\section{Structure analysis}

Structures and lattice defects were analyzed using OVITO [46] and the algorithms implemented therein. Crystalline ordering and its absence was identified using common neighbor analysis (CNA) with an adaptive cutoff $[48,49]$. Atomic volumes were obtained by Voronoi tessellation [50-53].

\section{RESULTS}

\section{A. Defect-free high-entropy alloys}

In the first step, we consider a defect-free HEA at ambient pressure. Figure 1(a) shows the VDOS $g$ as a function of the frequency $\nu$, both for the HEA and the glass
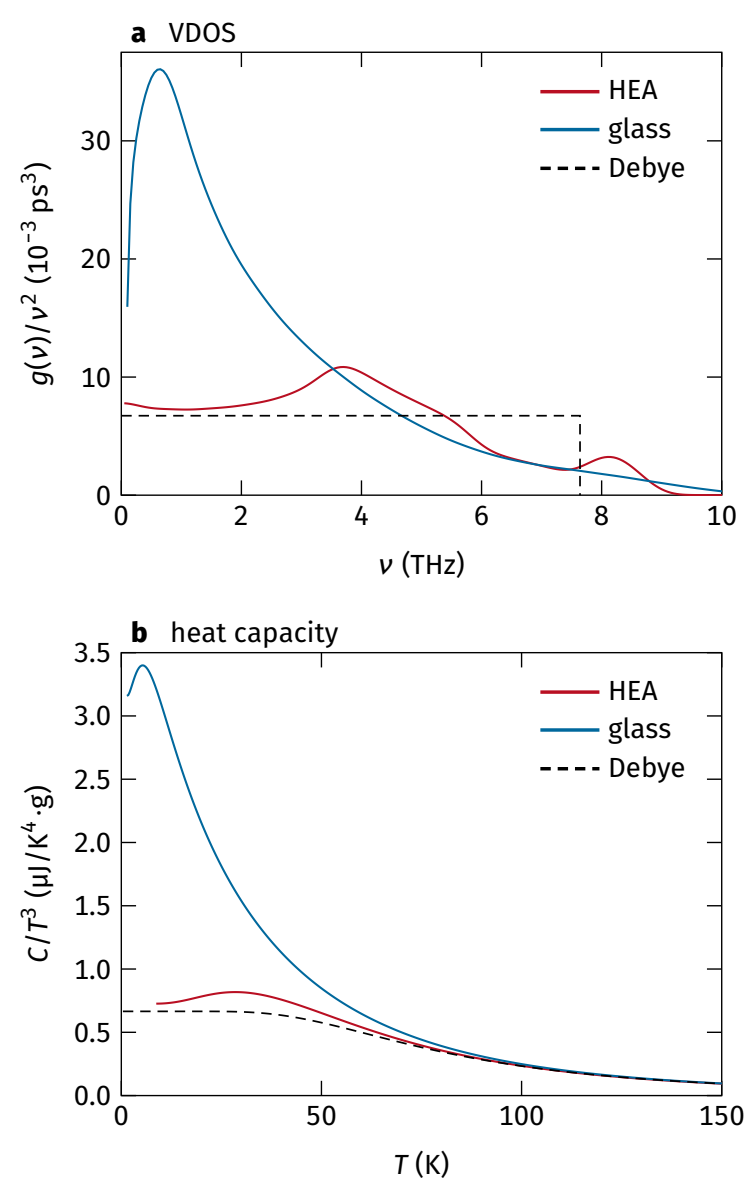

FIG. 1. Vibrational spectrum and heat capacity of a highentropy alloy compared with a glass. (a) Reduced VDOS of the CuNiCoFe high-entropy alloy and a glass with the same composition. A clear boson peak can be detected only in the glass; the high-entropy alloy simply exhibits a van Hove singularity at $4 \mathrm{THz}$. (b) The corresponding heat capacities as a function of temperature $T$. An excess between $30 \mathrm{~K}$ and $50 \mathrm{~K}$ is visible for the high-entropy alloy, which lies above the usual range for the boson peak of $10 \mathrm{~K}$ to $20 \mathrm{~K}$. 


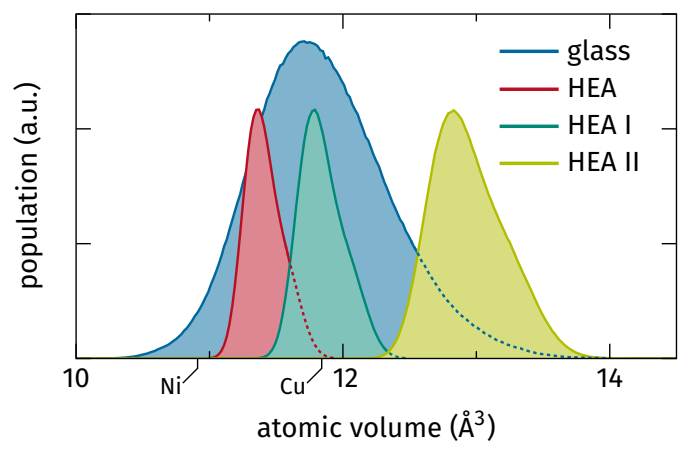

FIG. 2. Atomic volume distribution at $30 \mathrm{~K}$ of the highentropy alloy compared with a glass of the same composition. The equilibrium HEA (red) is denser than the glass (blue) and lies between the densities of pure fcc nickel and coppera small and a large constituent element of the alloy (indicated on the $x$ axis). The sample HEA I is deformed elastically to obtain the same average density as the glass, while HEA II was deformed elastically to obtain densities similar to the largest glass atoms.

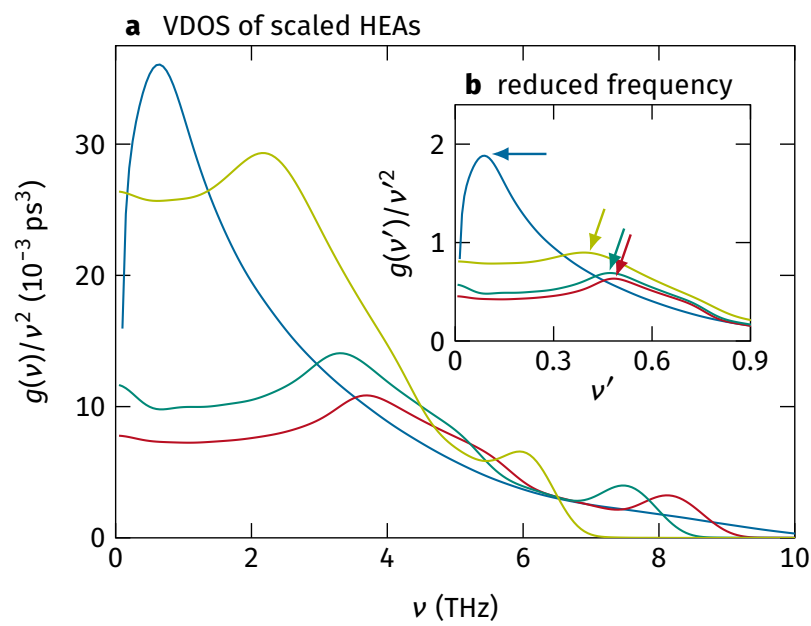

FIG. 3. (a) Reduced VDOS of the glass compared with the three high-entropy alloy samples of different densities. The color coding matches that in Fig. 2. The inset (b) shows the same as a function of the reduced frequency $\nu^{\prime}=\nu / \nu_{\mathrm{D}}$. These plots indicate that the van Hove singularity of the highentropy alloys cannot be shifted by varying the density to obtain a boson peak.

with the same composition. A reduced depiction $g(\nu) / \nu^{2}$ over $\nu$ is chosen in which the density of states postulated by the Debye model appears as a constant and any excess over it is easily visible. Figure $1(\mathrm{~b})$ contains the corresponding reduced heat capacities $C / T^{3}$. All curves feature an excess contribution. However, in the HEA the vibrational excess is located at around $4 \mathrm{THz}$, while the boson peak in the glass appears at the usual frequency of $1 \mathrm{THz}$.

Given recent theories that the boson peak is a densityshifted van Hove singularity and not disorder-related at all [30], it pays to have a more detailed look at the density dependence of the VDOS. Figure 2 contains an atomic volume histogram of the HEA and the glass: Clearly, the HEA has a higher density. In fact, the density of the HEA fluctuates between those of elemental $\mathrm{Ni}$ and elemental $\mathrm{Cu}$, a small and a large constituent element of the HEA. Consequently, we first prepared a sample with a similar density to the glass (HEA I). This was done by a simple, uniform scaling of the lattice to reach the desired density followed by constant-volume relaxation at $30 \mathrm{~K}$. Recent computer simulations on $\mathrm{Cu}-\mathrm{Zr}$-based metallic glasses demonstrate that only the atoms with the largest mean square displacement-which make up $10 \%$ of all atoms - contribute to the boson peak [12]. Assuming that those are the ones with the highest atomic volume, we also prepared a sample which covers atomic volumes among the highest in the glass (HEA II). The resulting VDOS measurements in Fig. 3(a) reveal that the glass VDOS is not reproduced even with a highly strained HEA. While the boson peak is found (as usual) in the $1 \mathrm{THz}$ region, the HEA peak only shifts to around $2 \mathrm{THz}$. Additionally, if plotting the reduced VDOS as a function of a reduced frequency $\nu^{\prime}=\nu / \nu_{\mathrm{D}}$, where $\nu_{\mathrm{D}}$ is the Debye frequency, the HEA peaks fall more or less on top of each other while the boson peak in the glass remains at lower $\nu^{\prime}$ [Fig. 3(b)].

Thus, comparing the VDOS of various strained and unstrained HEAs with a glass of the same composition does not provide evidence for a relation of the boson peak to a shifted van Hove singularity in our model alloy. Moreover, the data suggests that the chemical disorder of an HEA is insufficient to induce a boson peak. This does not exclude that lattices with stronger chemical disorder would show a boson peak; indeed, several theoretical investigations use fluctuations on a lattice to obtain these vibrational modes (see for example Ref. 18). Such increased disorder - which would most likely have to include elements with large size differences - is expected to destabilize the simple fcc, bcc, or hcp lattices of random metallic solid solutions [37, 54] and is therefore not realizable in single-phase HEAs.

\section{B. Introduction of disorder via interstitial defects}

In the next step, we consider the influence of defects on the vibrational spectrum since the boson peak was related to the lattice dynamics of defects [8] or loosely packed atoms $[6,10,12]$ and subatomic voids [13] in the past. Interstitialcy theory states that melting can be understood as the generation of an increasing number of interstitials, and that, consequently, glasses are also characterized by interstitial-like features [38]. Following this, we chose to randomly introduce interstitials into the HEA while keeping the composition equimolar. After the insertion of various concentrations of interstitials followed by equilibration at $30 \mathrm{~K}$ and ambient pressure, we obtained different structures: samples with $0.5 \%$ and 

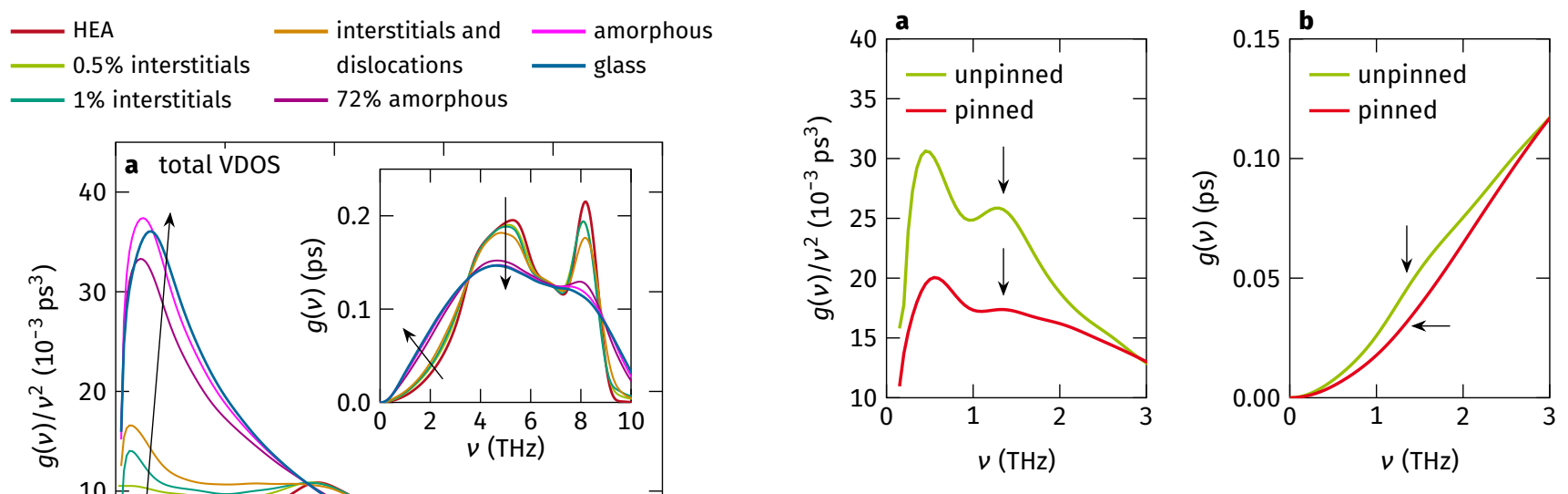

FIG. 5. The origin of the split boson peak for the sample with $0.5 \%$ of interstitials: When pinning low-volume atoms $\left(\Omega<10.5 \AA^{3}\right)$ which appear after the insertion of interstitials, the second peak is suppressed. This suggests that the splitting is a density-related frequency shift. The plots show the partial VDOS of only the defect atoms.

$1 \%$ interstitials, one sample in which most of the point defects collapsed into stacking faults and dislocation-like defects, one sample in which $72 \%$ of atoms where amorphous, and one sample which collapsed completely into an amorphous structure with small crystalline clusters of a few atoms. The defects were identified using CNA $[48,49]$ in OVITo [46].

This increasing disorder now indeed leads to a boson peak as shown in Fig. 4(a), where the peak height rises with the defect concentration. Contrary to other theoretical investigations (for example Ref. 20), the rise of this peak is not correlated with a shift of the boson peak towards lower frequencies. Instead, its position is more or less constant and even shifts to somewhat higher frequencies when the lattice collapses. We split the VDOS into a partial vibrational density of states (PDOS) for defective atoms surrounding point defects and atoms inside stacking faults and amorphous regions [Fig. 4(b)], and a PDOS for atoms on intact fcc lattice sites [Fig. 4(c)]. The interstitials and surrounding atoms already possess an almost full boson peak in the PDOS even at small concentrations. The contribution to the total VDOS is small only because the intact fcc lattice sites, which are in the majority, do not participate in the low-frequency modes. The frequency shift is even less pronounced in this plot. The fact that the boson peak is mostly absent in the PDOS of the intact lattice, especially at low defect concentrations, demonstrates its localized nature. This also explains the frequency of the boson peak modes: The only difference between the samples is the amount of defective areas. The disorder inside these areas is comparable and therefore the boson peak modes are located at the same frequencies. The inset of Fig. 4(a) explains the additional small frequency shift of the samples which were at least partially amorphous: The features of the crystalline VDOS smear out upon amorphization and the low-frequency tail 


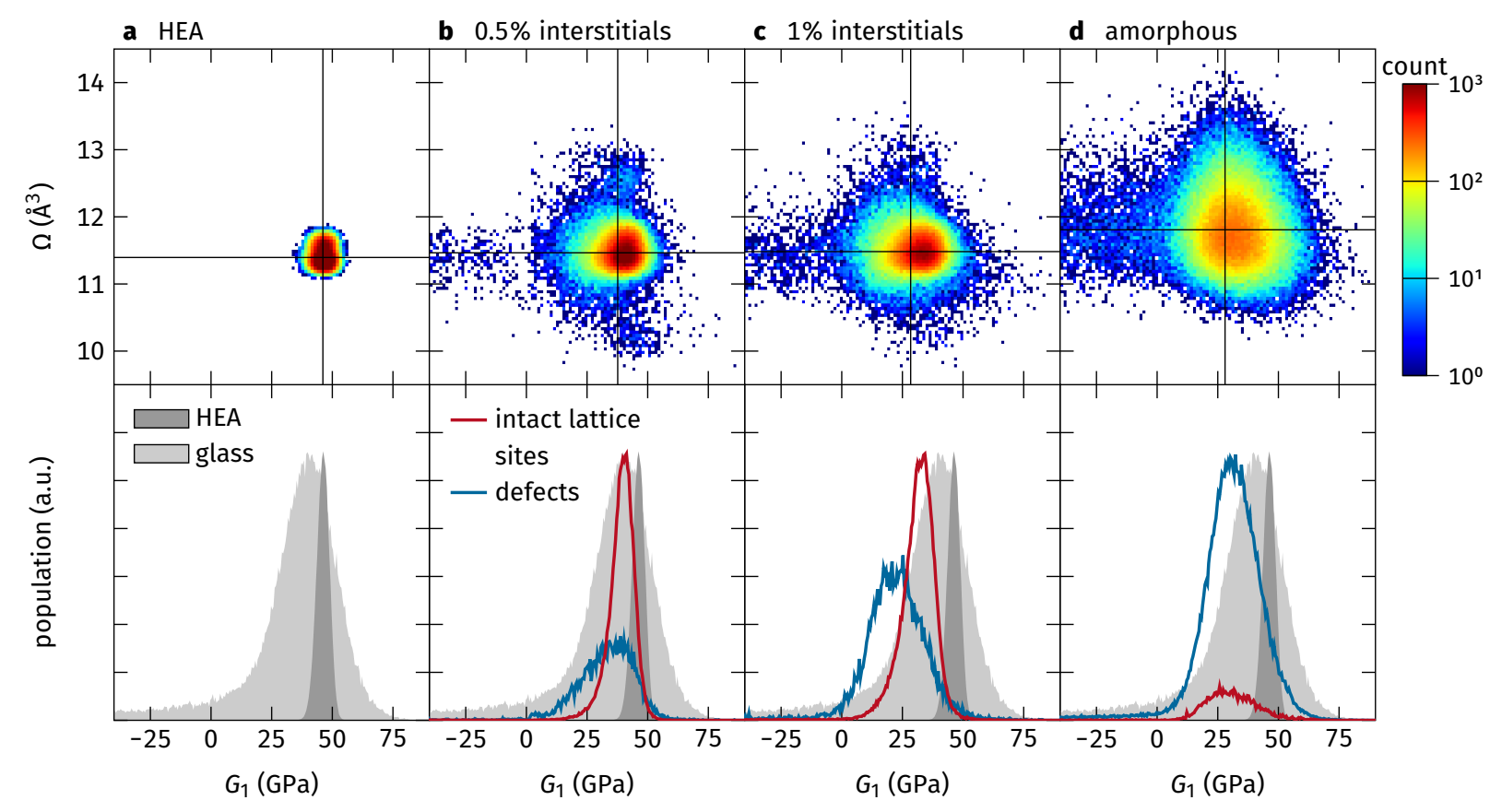

FIG. 6. Histograms of atomic volume and per-atom shear moduli: (a) defect-free HEA; (b) HEA with 0.5\% interstitials; (c) HEA with $1 \%$ interstitials; (d) amorphous system. The upper row shows $2 \mathrm{D}$ histograms of both the shear moduli $G_{1}$ and the atomic volumes $\Omega$ with a logarithmic color scale, while the lower row contains only the histograms of the shear moduli divided into defective atoms and atoms on intact fcc lattice sites. The horizontal and vertical lines in the upper row represent the average values. The gray areas in the lower row show the distributions of the reference glass, as well as the defect-free HEA. The relative height of the distributions is not to scale in order to make small amounts of defective or lattice atoms visible. The HEA in (a) shows a narrow distribution on both axes. (b)-(d) With increasing defect concentration, regions with shear moduli below $25 \mathrm{GPa}$ emerge. Those are due to the defect atoms, which have a shear modulus distribution similar to a bulk glass. (b) At low interstitial concentrations, atoms with increased and decreased volume appear. The decreased volume is obviously due to the insertion of interstitials, while the increased volume is unexpected. In fact, with increasing disorder in (c) and (d), the "compressed" atoms disappear. The reason for this is that the interstitials in the HEA always lead to local re-arrangements of the surrounding atoms, which in turn leads to a distribution of atomic volumes. The more the sample is defective, the easier the compressed atoms can release their stress and be accommodated in the disturbed lattice.

of the first crystalline peak overlaps with the boson peak, leading to an apparent shift to higher frequencies.

At $0.5 \%$ of interstitials, a second peak slightly below $2 \mathrm{THz}$ appears. It stands to reason that the introduction of interstitials leads to a compression of some neighboring atoms. While a chemically ordered system would only exhibit compressed atoms, the HEA locally rearranges and produces both atoms with increased and reduced atomic volume. Such a density difference could lead to a frequency shift between both types of defective atoms, which would in turn lead to a splitting of the boson peak. We tested this by pinning high-density atoms and recomputing the VDOS of the non-pinned defect atoms. Figure 5 shows that this indeed switches off the $2 \mathrm{THz}$ peak. At higher defect concentrations the split disappears. As we will discuss later in some more detail, the compressed atoms disappear with increasing defect concentration, as they are accommodated in the defective parts of the lattice.

The defect PDOS of the sample which contains both interstitials and dislocations seems to be reduced again, which indicates that the dislocation and stacking-fault defects do not contribute to the boson peak, thereby reducing the combined value.

Finally, the collapsed lattice has a VDOS similar to the glass, except for a small shift to lower frequencies. This can be explained by the fact that the collapsed glass did not have time for structural relaxation. This relaxation would reduce the density and consequently lead to a slight shift to higher frequencies. In the samples with such a high number of defects, the PDOS of the intact lattice sites also exhibit low-frequency modes. This is because a large fraction of these atoms has disordered neighbors, the vibrations of which also affect the fcc-ordered atoms. Still, even small fcc clusters resist a complete intrusion of the boson peak.

These results support the view that the boson peak is due to localized defect-related modes. A shift of a van Hove singularity can now be completely excluded as even the defect atoms still retain the VDOS shape of the crystal, although heavily smeared out. The boson peak arises as additional modes around the interstitials. 

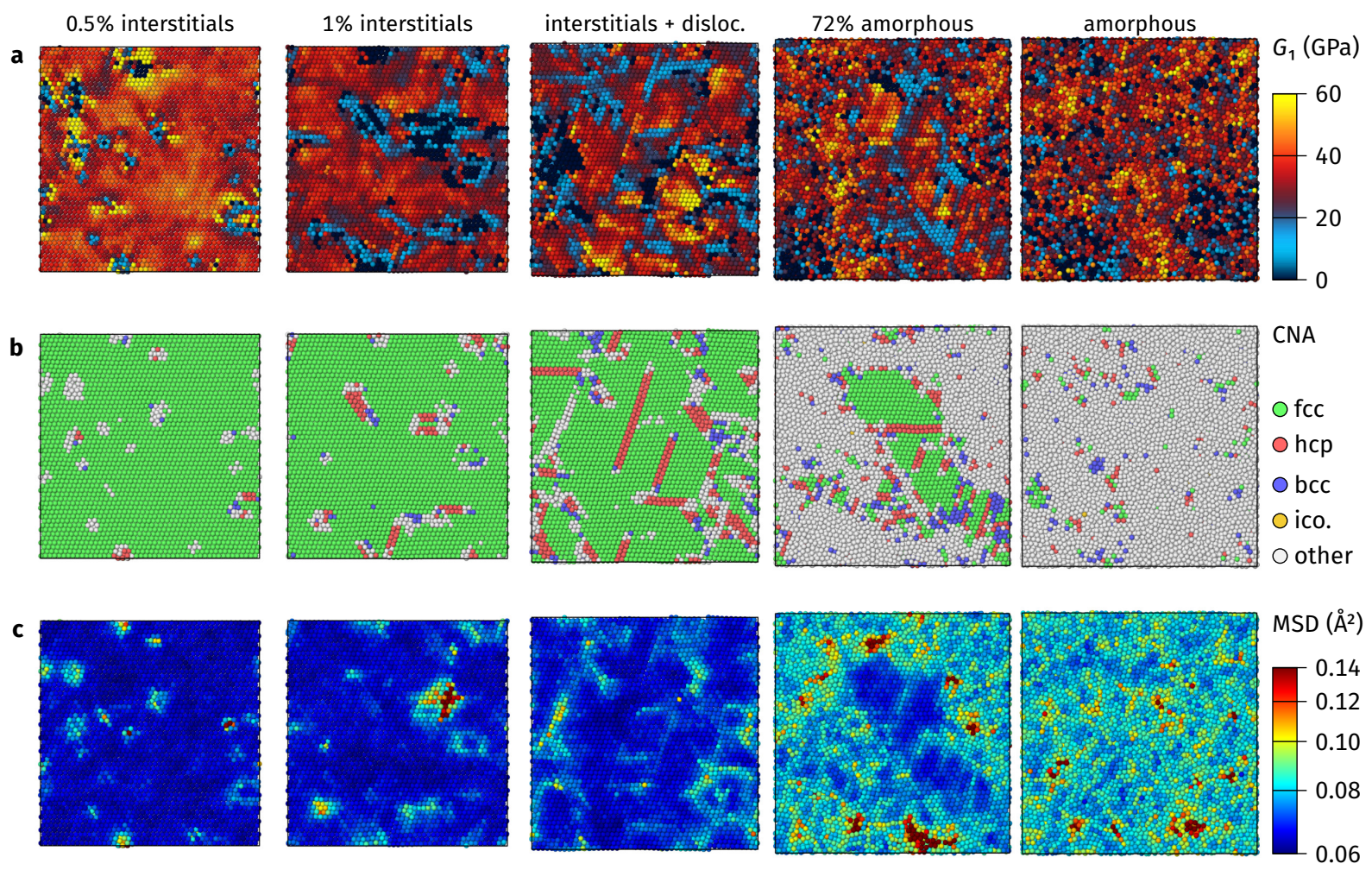

FIG. 7. Snapshots of samples with different defects, mapping the spatial distribution of shear modulus, disorder, and mean square displacement. A correlation between low shear modulus (a), defects as identified by CNA (b), and high mean square displacement (c) is apparent. The color scale in (c) is logarithmic to enhance the visibility of small displacements.

\section{Connection between defective structures and softening}

While we excluded that the fluctuations of elastic constants of the HEA are sufficient to induce a boson peak, it pays to have another look at the shear moduli of the defective samples. Following Derlet et al., we use the lowest of the five Kelvin shear moduli, $G_{1}$, which has been related to the boson peak [24]. Two-dimensional (2D) histograms of the atomic volume $\Omega$ versus the per-atom shear modulus $G_{1}$ are presented in Fig. 6. Although the HEA has fluctuations in both, they are small compared to the defective samples. Already at low interstitial concentrations, significant amounts of atoms with moduli between $0 \mathrm{GPa}$ and $25 \mathrm{GPa}$ appear. With increasing defect density the atoms with low modulus become more numerous. Thus, significant softening is needed for the boson peak, if it in fact results from fluctuating moduli. One can observe a general softening of the whole material with rising interstitial concentration, which points to a generally increasing instability of the material. This is to be expected at such high defect concentrations. The amorphous system is slightly softer than the quenched glass, which supports our earlier argument that the quenched glass represents a more relaxed state. Qualitatively, the two systems are similar. Figure 6(b) also proves our earlier statement that atoms with increased as well as re- duced density occur. As we can see in Figs. 6(c) and 6(d), the unfavorable high-density atoms start disappearing because the systems with higher defect concentrations can re-arrange more easily. With them, the split in the boson peak also disappears.

The remaining question is, if the defect-based boson peak models are indeed as different as they initially appear to be. Studies ascribe the boson peak to interstitialcies [14, 15], "liquidlike" regions [13, 25], "rattling" atoms [11, 12], and of course fluctuating force constants [17-19, 21, 22]. So, in addition to local shear moduli, we calculated the mean square displacement (MSD) of the atoms at $30 \mathrm{~K}$ over a period of $100 \mathrm{ps}$. Shear modulus $G_{1}$, structure as identified by CNA, and MSD are shown in Fig. 7. The spatial correlation between defect atoms, low shear modulus, and high MSD is apparent. This is not surprising: The defects introduced into the lattice are well known to soften the material [38]. Furthermore, low moduli are of course connected with shallow potential wells and therefore a high MSD. This suggests that softened regions can be introduced by interstitials, and that these regions resemble the "soft spots" discussed in the literature on metallic glasses $[13,25]$. Thus, previous studies seem to observe the same effect by measuring different quantities. 


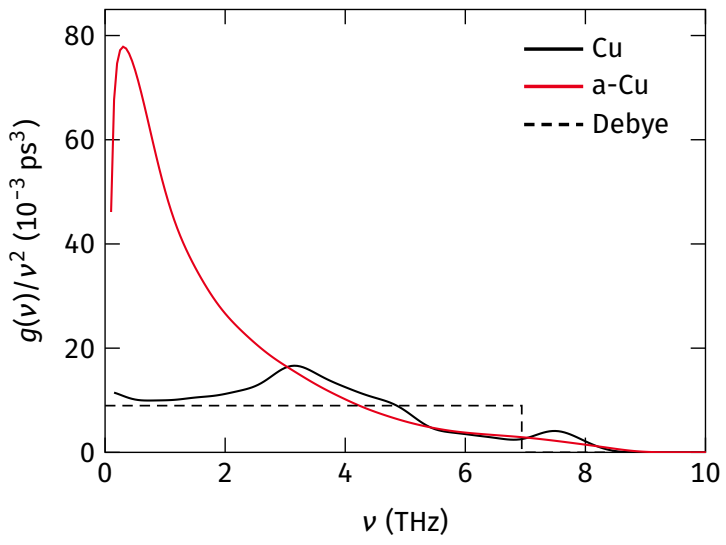

FIG. 8. Comparison of the VDOS of fcc copper with amorphous copper. A boson peak occurs even without chemical disorder. The VDOS of crystalline copper resembles that of the HEA and is typical for fcc metals. The peak around $3.5 \mathrm{THz}$ is a van Hove singularity.

\section{A boson peak without chemical disorder}

This analysis again supports the view that the loss of structural order is the key ingredient for obtaining the boson peak. In order to supplement this picture, we went one step further and removed the chemical disorder from the model structure by studying a single-component glass. We repeated the analysis presented in Fig. 1 for fcc and amorphous copper $(\mathrm{a}-\mathrm{Cu})$ quenched from the melt with $10^{14} \mathrm{~K} / \mathrm{s}$. As can be seen in Fig. 8, the a-Cu sample also exhibits an excess of states at low frequencies. This evidences that chemical disorder is not only not a sufficient condition for the boson peak, but that it is not even a necessary one. Indeed, the shape of the VDOS of fcc copper resembles that of the HEA. The excess states in the HEA are simply due to a van Hove singularity. Contrary to recent theories [30-34], this van Hove singularity is not the same as a boson peak. As demonstrated above, (i) the van Hove singularity does not shift to $1 \mathrm{THz}$ when scaling the density to values typical for the glass, and (ii) the crystalline features of the VDOS also appear in the glass; heavily smeared out but unshifted.

\section{CONCLUSION}

All in all, the current work supports the conclusion that the boson peak in alloys results from (quasi-)localized ad- ditional modes. The vibrational modes of interstitial defects resemble those of a glass and the atomic volumes around those defects do not exceed the values in HEA II, a defect-free lattice with reduced density. This excludes a simple density-related origin of these additional modes in the system we considered, since HEA II did not exhibit a boson peak. In fact, the resemblance of the PDOS in Fig. 4(b) to the crystalline state excludes a general shift of frequencies. This is also in accordance with recent measurements and simulations of the boson peak in deformed $\mathrm{Cu}-\mathrm{Zr}$ and $\mathrm{Pd}-\mathrm{Ni}-\mathrm{P}$ glasses $[55,56]$. The boson peak contribution inside the shear band was found to be higher than in the undeformed material. While one could argue that the density in the shear band is lowered $[57,58]$, the boson peak does not shift very much on the frequency or temperature axis. Together with the current results, and the fact that we know that short-range order inside the shear bands is disturbed [57, 58], this data rather supports the defect picture outlined above. The results presented here also confirm what was suggested by Schirmacher and colleagues [22]: Defect-based pictures of the boson peak origin can be united with the fluctuating force-constant model, although our results indicate that a significant localized softening is needed. The "soft spot" picture of metallic glasses seems relevant here: Regions of defective short-range order are connected with lower stiffness and strength as well as with a high boson peak signal [13, 23-25]. Of course, atoms in these regions exhibit high MSDs as was shown in previous studies $[11,12]$. This suggests a relevance of the boson peak not only in the realm of solid-state physics, but also for the mechanical properties of metallic glasses.

\section{ACKNOWLEDGMENTS}

The authors thank Alexander Stukowski for help with Ovito, Peter Derlet for pointing us to the Kelvin stiffness tensor, as well as Gerhard Wilde and Sergiy Divinski for discussions about the low-temperature heat capacity of HEAs. Financial support by the Deutsche Forschungsgemeinschaft (DFG) through Grant No. AL 578/6-2, as well as a travel grant to Finland through the PPP program of the Deutscher Akademischer Austauschdienst (DAAD) are gratefully acknowledged. Computing time was made available by the Technische Universität Darmstadt on the Lichtenberg cluster.
* brink@mm.tu-darmstadt.de

1 S. R. Elliott, EPL 19, 201 (1992).

${ }^{2}$ V. G. Karpov, M. I. Klinger, and F. N. Ignat'ev, Zh. Eksp. Teor. Fiz. 84, 760 (1983).
3 B. B. Laird and H. R. Schober, Phys. Rev. Lett. 66, 636 (1991).

${ }^{4}$ H. R. Schober and B. B. Laird, Phys. Rev. B 44, 6746 (1991). 
5 L. Gil, M. A. Ramos, A. Bringer, and U. Buchenau, Phys. Rev. Lett. 70, 182 (1993).

${ }^{6}$ H. R. Schober and C. Oligschleger, Phys. Rev. B 53, 11469 (1996).

7 H. Shintani and H. Tanaka, Nat. Mater. 7, 870 (2008).

${ }^{8}$ H. R. Schober, J. Non-Cryst. Solids 357, 501 (2011).

${ }^{9}$ H. R. Schober, U. Buchenau, and V. L. Gurevich, Phys. Rev. B 89, 014204 (2014).

${ }^{10}$ Y. Li, H. Y. Bai, W. H. Wang, and K. Samwer, Phys. Rev. B 74, 052201 (2006).

11 M. Guerdane and H. Teichler, Phys. Rev. Lett. 101, 065506 (2008).

12 N. Jakse, A. Nassour, and A. Pasturel, Phys. Rev. B 85, 174201 (2012).

13 H. Sheng, E. Ma, and M. Kramer, JOM 64, 856 (2012).

14 A. V. Granato, Physica B: Condens. Matter 219-220, 270 (1996).

15 A. N. Vasiliev, T. N. Voloshok, A. V. Granato, D. M. Joncich, Y. P. Mitrofanov, and V. A. Khonik, Phys. Rev. B 80, 172102 (2009).

16 T. S. Grigera, V. Martín-Mayor, G. Parisi, and P. Verrocchio, Nature 422, 289 (2003).

17 W. Schirmacher and M. Wagener, Solid State Commun. 86, 597 (1993).

18 W. Schirmacher, G. Diezemann, and C. Ganter, Phys. Rev. Lett. 81, 136 (1998).

19 W. Schirmacher, G. Ruocco, and T. Scopigno, Phys. Rev. Lett. 98, 025501 (2007).

${ }^{20}$ W. Schirmacher, B. Schmid, C. Tomaras, G. Viliani, G. Baldi, G. Ruocco, and T. Scopigno, Phys. Status Solidi C 5, 862 (2008).

21 A. Marruzzo, W. Schirmacher, A. Fratalocchi, and G. Ruocco, Sci. Rep. 3, 1407 (2013).

${ }^{22}$ W. Schirmacher, T. Scopigno, and G. Ruocco, J. NonCryst. Solids 407, 133 (2015).

23 A. Tanguy, B. Mantisi, and M. Tsamados, EPL 90, 16004 (2010).

24 P. M. Derlet, R. Maaß, and J. F. Löffler, EPJ B 85, 148 (2012).

25 J. Ding, S. Patinet, M. L. Falk, Y. Cheng, and E. Ma, Proc. Natl. Acad. Sci. USA 111, 14052 (2014).

${ }^{26}$ F. Léonforte, A. Tanguy, J. P. Wittmer, and J.-L. Barrat, Phys. Rev. Lett. 97, 055501 (2006).

27 C. Fusco, T. Albaret, and A. Tanguy, Phys. Rev. E 82, 066116 (2010).

${ }^{28}$ Y. M. Beltukov, C. Fusco, D. A. Parshin, and A. Tanguy, Phys. Rev. E 93, 023006 (2016).

29 S. N. Taraskin, Y. L. Loh, G. Natarajan, and S. R. Elliott, Phys. Rev. Lett. 86, 1255 (2001).

30 A. I. Chumakov, G. Monaco, A. Monaco, W. A. Crichton, A. Bosak, R. Rüffer, A. Meyer, F. Kargl, L. Comez, D. Fioretto, H. Giefers, S. Roitsch, G. Wortmann, M. H. Manghnani, A. Hushur, Q. Williams, J. Balogh, K. Parliński, P. Jochym, and P. Piekarz, Phys. Rev. Lett. 106, 225501 (2011).

31 A. I. Chumakov, G. Monaco, A. Fontana, A. Bosak, R. P. Hermann, D. Bessas, B. Wehinger, W. A. Crichton,
M. Krisch, R. Rüffer, G. Baldi, G. Carini Jr., G. Carini, G. D'Angelo, E. Gilioli, G. Tripodo, M. Zanatta, B. Winkler, V. Milman, K. Refson, M. T. Dove, N. Dubrovinskaia, L. Dubrovinsky, R. Keding, and Y. Z. Yue, Phys. Rev. Lett. 112, 025502 (2014).

32 A. I. Chumakov and G. Monaco, J. Non-Cryst. Solids 407, 126 (2015).

33 A. I. Chumakov, G. Monaco, X. Han, L. Xi, A. Bosak, L. Paolasini, D. Chernyshov, and V. Dyadkin, Philos. Mag. 96, 743 (2016).

34 G. Baldi, G. Carini Jr., G. Carini, A. Chumakov, R. D. Maschio, G. D'Angelo, A. Fontana, E. Gilioli, G. Monaco, L. Orsingher, B. Rossi, and M. Zanatta, Philos. Mag. 96 , 754 (2016).

35 J.-W. Yeh, JOM 65, 1759 (2013).

36 M.-H. Tsai and J.-W. Yeh, Mater. Res. Lett. 2, 107 (2014).

37 D. B. Miracle, J. D. Miller, O. N. Senkov, C. Woodward, M. D. Uchic, and J. Tiley, Entropy 16, 494 (2014).

38 V. A. Khonik, Metals 5, 504 (2015).

39 X. W. Zhou, R. A. Johnson, and H. N. G. Wadley, Phys. Rev. B 69, 144113 (2004).

40 S. Plimpton, J. Comp. Phys. 117, 1 (1995), http:// lammps.sandia.gov/.

41 B. Sadigh, P. Erhart, A. Stukowski, A. Caro, E. Martinez, and L. Zepeda-Ruiz, Phys. Rev. B 85, 184203 (2012).

42 A. G. Wilson, Covariance kernels for fast automatic pattern discovery and extrapolation with Gaussian processes, Ph.D. thesis, Trinity College, University of Cambridge (2014).

43 J. M. Dickey and A. Paskin, Phys. Rev. 188, 1407 (1969).

44 R. K. Pathria, Statistical Mechanics, 2nd ed. (Elsevier Butterworth-Heinemann, Oxford, 1996).

${ }^{45}$ F. Shimizu, S. Ogata, and J. Li, Mater. Trans. 48, 2923 (2007).

46 A. Stukowski, Model. Simul. Mater. Sci. Eng. 18, 015012 (2010), http://ovito.org/.

47 W. Thomson, Philos. Trans. Roy. Soc. London 146, 481 (1856).

48 J. D. Honeycutt and H. C. Andersen, J. Phys. Chem. 91, 4950 (1987).

49 A. Stukowski, Model. Simul. Mater. Sc. 20, 045021 (2012).

50 G. Voronoï, J. Reine Angew. Math. 133, 97 (1908).

51 G. Voronoï, J. Reine Angew. Math. 134, 198 (1908).

52 G. Voronoï, J. Reine Angew. Math. 136, 67 (1909).

${ }^{53}$ W. Brostow, M. Chybicki, R. Laskowski, and J. Rybicki, Phys. Rev. B 57, 13448 (1998).

54 A. Inoue, Acta Mater. 48, 279 (2000).

55 J. Bünz, T. Brink, K. Tsuchiya, F. Meng, G. Wilde, and K. Albe, Phys. Rev. Lett. 112, 135501 (2014).

56 Y. P. Mitrofanov, M. Peterlechner, S. V. Divinski, and G. Wilde, Phys. Rev. Lett. 112, 135901 (2014)

57 Y. Ritter and K. Albe, Acta Mater. 59, 7082 (2011).

58 Y. Ritter and K. Albe, J. Appl. Phys. 111, 103527 (2012). 